\title{
Long axis excursion in aortic stenosis
}

\author{
S Takeda, H Rimington, N Smeeton, J Chambers
}

\begin{abstract}
Objectives-To examine long axis excursion in patients with all grades of aortic stenosis and preserved transverse systolic function, and to compare long axis excursion in symptomatic with that in asymptomatic severe aortic stenosis.

Design-Prospective comparative study.

Setting-Regional cardiothoracic centre.

Patients-78 patients with all grades of aortic stenosis and normal fractional shortening and ejection fraction were studied. There were two comparison groups, 10 age matched normal subjects and 14 patients with aortic stenosis and fractional shortening $<26 \%$.

Methods-Aortic valve function and left ventricular mass were assessed echocardiographically. $M$ mode measurements of long axis excursion at the septal and lateral sides of the mitral annulus were taken.

Results-There were significant differences between the groups in long axis excursion at both the septal $(p<0.0001)$ and lateral sides of the mitral annulus $(p=0.002$ by analysis of variance). Long axis excursion was independently related to both left ventricular mass index $(p=0.001)$ and the grade of aortic stenosis $(\mathrm{p}=0.002)$. Comparing patients with severe aortic stenosis with and without symptoms, there were significant differences in effective orifice area $(p=0.02)$ and long axis excursion at the lateral side of the mitral annulus $(p=0.04)$, but not in fractional shortening, ejection fraction, or peak or mean pressure difference.

Conclusion-In patients with aortic stenosis, long axis excursion is reduced even in the presence of normal fractional shortening or ejection fraction. It is lower in patients with symptomatic compared with asymptomatic severe aortic stenosis and may be of use in predicting the onset of symptoms.
\end{abstract}

(Heart 2001;86:52-56)

Keywords: aortic stenosis; long axis excursion; symptoms

Conservative management is usually recommended for haemodynamically severe but asymptomatic aortic stenosis. ${ }^{1-3}$ However, sudden death may occur and the mortality in the first few weeks after the onset of symptoms is around $10 \% .{ }^{145}$ By comparison the surgical mortality rate may be as low as $2 \%$ for fit young subjects having elective surgery. ${ }^{6}$ A reliable method of predicting the onset of symptoms might allow the consideration of prophylactic investigation and even surgery with the prospect of saving lives. Current methods of predicting symptoms are based on the haemodynamic assessment of the aortic stenosis, ${ }^{7}$ and there is surprisingly little information on the effect of left ventricular function. Measures of left ventricular function are known to deteriorate as aortic stenosis progresses, ${ }^{89}$ and there is an association between the presence of symptoms and minor transverse systolic dysfunction. ${ }^{10}$ Long axis excursion may provide an earlier warning of the onset of symptoms.

Long axis excursion is a measure of the function of longitudinally or spirally arranged fibres in the subendocardial and subepicardial layers of the myocardium that are responsible for the ventricle becoming more spherical during isovolumetric contraction. The larger intermediate layer of transversely arranged fibres causes ejection of blood during systole. The subendocardial fibres are at greater risk from ischaemia than the intermediate layers so that longitudinal dysfunction precedes transverse dysfunction in patients with coronary artery disease, ${ }^{11}$ hypertension, mitral valve disease, and dilated cardiomyopathy. ${ }^{12-14}$ Long axis dysfunction has also been shown in patients with aortic stenosis and fractional shortening $<25 \%{ }^{15}$ However, patients with preserved transverse function have not been studied.

The aims of our study were to compare long axis excursion in patients with all grades of aortic stenosis and preserved transverse systolic function, and to see whether long axis excursion differed between symptomatic and asymptomatic patients with severe aortic stenosis.

\section{Methods}

PATIENTS

Consecutive patients were recruited from the echocardiography department on three study days each week from 1 May 1996 to 31 May 1998. A total of 99 patients met the inclusion criteria of aortic valve thickening, peak instantaneous transaortic velocity $>2.5 \mathrm{~m} / \mathrm{s}$ and normal systolic function defined by fractional shortening $>28 \%$, and the absence of a resting wall motion abnormality. Exclusions were for suboptimal image quality $(n=3)$, coexistent mitral valve disease $(n=4)$, more than mild aortic regurgitation $(n=6)$, pericardial effusion $(n=1)$, and incomplete data $(n=7)$. There remained 78 patients, mean (SD) age 64 (13) years, of whom 46 (59\%) were men. Coronary angiography was performed in 36 patients at a mean of 3.7 (6.5) months before or after echocardiography based on routine clinical grounds and on the decision of the
Correspondence to:

johnchambers@dial.pipex.com

Accepted 21 December 2000 
physician in charge of the case without knowledge of long axis function. Significant coronary artery disease was defined by a stenosis $>70 \%$ of the luminal diameter estimated by eye in at least one epicardial artery and was found in 13 of the 36 patients. Twenty five patients reported significant symptoms: exertional chest pain in nine, syncope in four, and severe breathlessness in 12. Minor non-specific breathlessness was taken as normal. There were two comparison groups. One group comprised 14 subjects with aortic valve thickening similar to the study group but with systolic dysfunction defined by a fractional shortening $<26 \%$ in the absence of a regional wall motion abnormality, three of whom had significant coronary artery disease on coronary angiography. The other comparison group consisted of 10 age matched subjects recruited from relatives of patients who were attending for day case coronary angiography. None had a history of heart disease, hypertension, diabetes, or pulmonary or autoimmune disease. The study was approved by the local committee on ethical practice and all subjects gave written informed consent.

\section{ECHOCARDIOGRAPHY}

Echocardiography was performed using an ATL HDI 3000 (Seattle, Washington, USA) with a 3-2 $20 \mathrm{~mm}$ duplex probe and $1.9 \mathrm{MHz}$ continuous wave probe. Minor axis $M$ mode recordings were made at a level just below the tips of the mitral leaflets. End diastolic measurements were made according to the American Society of Echocardiography convention. ${ }^{16}$ Fractional shortening was calculated as the systolic decrease in left ventricular minor axis divided by end diastolic diameter. Left ventricular mass was estimated by the Troy formula ${ }^{17}$ and indexed to body surface area. Ejection fraction was estimated using visual assessment in all views ${ }^{18}$ aided by $M$ mode dimensions at the base of the heart. ${ }^{19}$ This was a subsidiary measure of systolic function for the purposes of comparison with the literature.

$M$ mode measurements of the left ventricular long axis were made from the apical four chamber view with the cursor positioned at the septal and lateral sides of the mitral valve annulus. Long axis excursion $(\mathrm{cm})$ at each site was taken from the nadir of the A wave, defined as the maximal backward displacement of the mitral

Table 1 Patient demographics

\begin{tabular}{lllll}
\hline & $\begin{array}{l}\text { Normal controls } \\
(n=10)\end{array}$ & $\begin{array}{l}\text { AS study group } \\
(n=78)\end{array}$ & $\begin{array}{l}\text { AS patients abnormal } \\
\text { LVEF }(n=14)\end{array}$ & $\begin{array}{l}p \text { Value (if } \leqslant \\
0.05 \text { by ANOVA) }\end{array}$ \\
\hline Age (years) & $63(5)$ & $64(13)$ & $71(9)$ & \\
Male & $2(20 \%)$ & $46(59 \%)$ & $5(38 \%)$ & 0.02 \\
CAD & $0 / 0$ & $13 / 36(36 \%)$ & $3 / 7(43 \%)$ & \\
Hypertension & 0 & $31(40 \%)$ & $2(14 \%)$ & $<0.0001$ \\
Mean PG (mmHg) & $2(1)$ & $37(18)$ & $46(26)$ & $<0.0001$ \\
Peak PG (mmHg) & $3(1)$ & $60(27)$ & $71(39)$ & $<0.0001$ \\
EOA $\left(\mathrm{cm}^{2}\right)$ & $2.3(0.5)$ & $0.9(0.3)$ & $0.7(0.2)$ & $<0.0001$ \\
FS $(\%)$ & $39(8)$ & $39(7)$ & $21(4)$ & 0.0001 \\
EF $(\%)$ & $77(9)$ & $76(7)$ & $49(9)$ & 0.0002 \\
LVMI $\left(\mathrm{g} / \mathrm{m}^{2}\right)$ & $69(29)$ & $152(58)$ & $177(84)$ &
\end{tabular}

Results are given as mean (SD) unless otherwise indicated.

${ }^{\star} \mathrm{CAD}$, coronary artery disease, showing number of patients with $\mathrm{CAD} /$ number of patients who underwent coronary angiography

ANOVA, analysis of variance; EF, ejection fraction; EOA, effective orifice area; FS, fractional shortening; LVEF, left ventricular ejection fraction; LVMI, left ventricular mass index; PG, transaortic pressure difference. annulus after the $\mathrm{P}$ wave, to the point of peak shortening. The subaortic diameter was measured on a parasternal long axis frame frozen in systole. An average of three estimates was taken from inner to inner edge. Pulsed Doppler recordings were made in the five chamber view with the sample volume moved axially from the level of the aortic annulus until a clear unaliased signal was obtained. The signal was planimetered to obtain peak velocity, velocity time integral, and mean pressure difference using the online software. Continuous wave recordings were made from the apex and right intercostal positions and the optimal signal was planimetered for peak velocity, velocity time integral, and mean pressure difference. Three pulsed or continuous wave signals were planimetered if the patient was in sinus rhythm and six to 10 if in atrial fibrillation.

Peak pressure difference ( $\mathrm{mm} \mathrm{Hg}$ ) was calculated using the long form of the modified Bernouilli equation: peak $\Delta \mathrm{P}=4\left(\mathrm{v}_{2}{ }^{2}-\mathrm{v}_{1}{ }^{2}\right)$, where $v_{1}$ is subaortic peak velocity $(\mathrm{m} / \mathrm{s})$ and $\mathrm{v}_{2}$ is aortic peak velocity $(\mathrm{m} / \mathrm{s})$. Effective orifice area $\left(\mathrm{EOA}, \mathrm{cm}^{2}\right)$ was calculated using the classical form of the continuity equation: $\mathrm{EOA}=\mathrm{CSA} \times \mathrm{vti}_{1} / \mathrm{vti}_{2}$, where CSA is cross sectional area of the left ventricular outflow tract $\left(\mathrm{cm}^{2}\right)$, and $\mathrm{vti}_{1}$ and $\mathrm{vti}_{2}$ are the subaortic and aortic velocity time integrals, respectively $(\mathrm{cm})$.

Intraobserver variability for long axis excursion was $5 \%$ and interobserver variability was $7 \%$.

\section{STATISTICAL ANALYSIS}

Values are presented as mean (SD). Patients with normal fractional shortening were divided according to the grade of aortic stenosis, as defined by EOA determined using the continuity equation, as mild $\left(\mathrm{EOA}>1.0 \mathrm{~cm}^{2}\right)$, moderate (EOA 0.75-1.0 $\mathrm{cm}^{2}$ ), and severe (EOA $<0.75 \mathrm{~cm}^{2}$ ). The five groups were compared by one way analysis of variance followed by Student-Newman-Keuls post hoc testing for pairs of groups. Long axis excursion was compared with left ventricular mass index using regression analysis, and with patients with and without coronary artery disease by an unpaired $t$ test.

No patient with mild aortic stenosis and only two of 22 with moderate aortic stenosis reported symptoms. However, of 34 patients with severe aortic stenosis, 23 had symptoms and these patients were compared by unpaired Student's $t$ tests with the remaining 11 asymptomatic patients according to age, left ventricular mass, long axis function, fractional shortening, ejection fraction, peak and mean pressure difference, and EOA. Symptomatic and asymptomatic patients were compared for the presence or absence of coronary artery disease by a $\chi^{2}$ test. A multivariate model was then constructed and logistic regression analysis used to determine independent predictors of symptoms.

\section{Results}

\section{GENERAL RESULTS}

Demographic data are shown in table 1 . The peak transaortic pressure difference was 60 

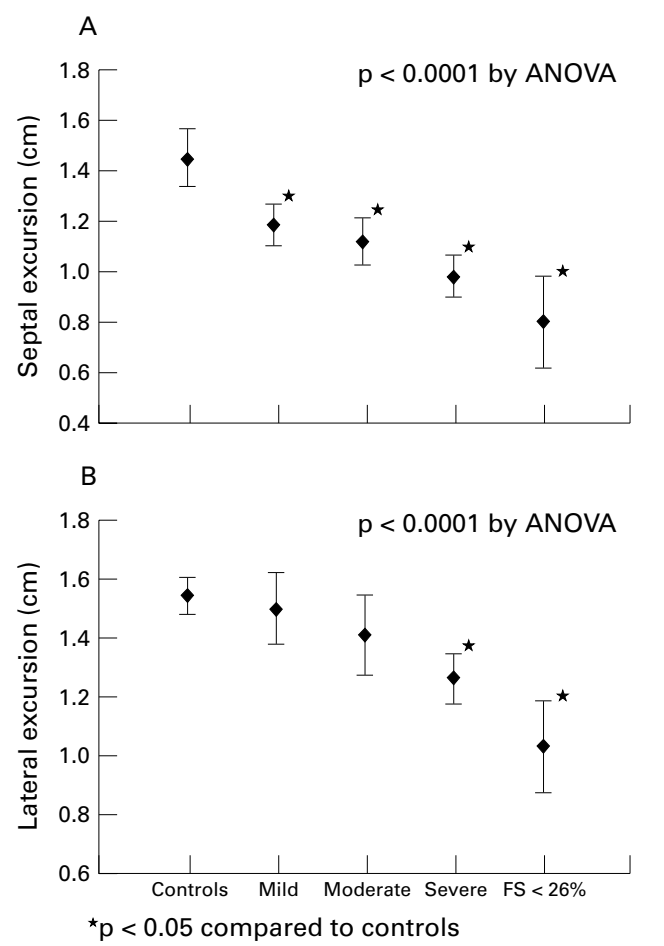

Figure 1 Long axis excursion (mean and 95\% confidence interval) for all grades of aortic stenosis and the two comparison groups. FS, fractional shortening

(27) $\mathrm{mm} \mathrm{Hg}$ (range $20-136 \mathrm{~mm} \mathrm{Hg}$ ) and the mean pressure difference 37 (18) $\mathrm{mm} \mathrm{Hg}$ (range 10-82 $\mathrm{mm} \mathrm{Hg}$ ). In 22 patients the aortic stenosis was mild, in 22 moderate, and in 34 severe as defined by the continuity equation.

LONG AXIS FUNCTION

There were significant differences in long axis excursion at both the septal $(\mathrm{p}<0.0001)$ and the lateral sides of the annulus $(p=0.002)$. For the septal side, the difference compared with the normal comparison group was significant for patients with all grades of aortic stenosis $(p<0.05)$. For the lateral side, the difference was significant only for patients with severe aortic stenosis $(p<0.05)$ (fig 1$)$. Long axis excursion was lower in the comparison group with impaired fractional shortening than in patients with all grades of aortic stenosis but normal fractional shortening.

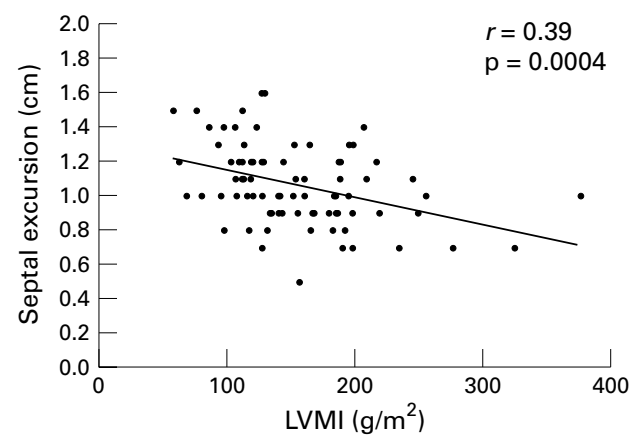

Figure 2 Linear regression for septal excursion of the mitral annulus $(\mathrm{cm})$ and left ventricular mass index (LVMI) $\left(g / \mathrm{m}^{2}\right)$.
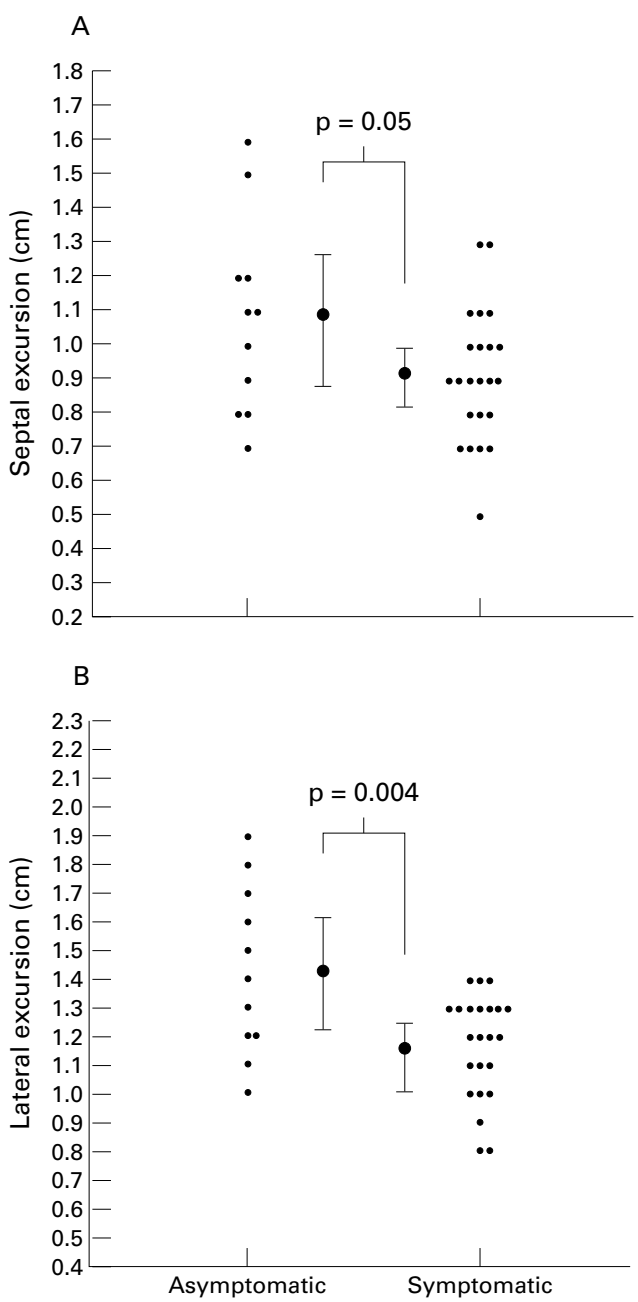

Figure 3 Long axis excursion at septal and lateral sides of the mitral annulus. The mean and 95\% confidence intervals are shown.

RELATION BETWEEN LONG AXIS FUNCTION AND LEFT VENTRICULAR MASS OR CORONARY ARTERY DISEASE

There was a significant linear relation between long axis excursion and left ventricular mass index at the septal side $(y=-0.0016 x+1.31$, $\mathrm{p}<0.0004$ ) (fig 2) but not at the lateral side of the mitral annulus $(y=-0.00099 x+1.51$, $\mathrm{p}=0.08)$. On multivariate analysis, long axis excursion at the septal side was independently related to both left ventricular mass index $(p=0.001)$ and the grade of aortic stenosis $(\mathrm{p}=0.002)$.

In those patients with and those without coronary disease, there was no significant difference in long axis excursion at the septal side $(1.07(0.23) \mathrm{cm} v 1.07(0.24) \mathrm{cm}$, $\mathrm{p}=0.92)$ or the lateral side of the mitral annulus $(1.34(0.23) \mathrm{cm}$ v $1.37(0.30) \mathrm{cm}$, $\mathrm{p}=0.79)$.

\section{SYMPTOMATIC COMPARED WITH ASYMPTOMATIC} PATIENTS WITH SEVERE AORTIC STENOSIS

There were 23 patients with severe aortic stenosis who had symptoms and 11 who were asymptomatic. There were no significant differences in peak transaortic pressure difference (85 (23) mm Hg $v 76$ (12) mm Hg, p = 0.19), 
Table 2 Severe aortic stenosis: asymptomatic compared with symptomatic patients

\begin{tabular}{llll}
\hline & $\begin{array}{l}\text { Asymptomatic } \\
(n=11)\end{array}$ & $\begin{array}{l}\text { Symptomatic } \\
(n=23)\end{array}$ & $\begin{array}{c}p \text { Value } \\
\text { (if } \leqslant 0.05)\end{array}$ \\
\hline Age (years (SD)) & $67(15)$ & $65(10)$ & \\
Sex (male) & $7(64 \%)$ & $9(39 \%)$ & \\
Mean pressure difference (mm Hg) & $47(9)$ & $54(14)$ & \\
Peak pressure difference (mm Hg) & $76(12)$ & $85(22)$ & \\
Effective orifice area $\left(\mathrm{cm}^{2}\right)$ & $0.70(0.07)$ & $0.58(0.12)$ & 0.006 \\
LVMI $\left(\mathrm{g} / \mathrm{m}^{2}\right)$ & $163(57)$ & $159(67)$ & \\
Ejection fraction $(\%)$ & $77(8)$ & $75(8)$ & \\
Fractional shortening $(\%)$ & $39(8)$ & $38(7)$ & \\
Septal excursion $(\mathrm{cm})$ & $1.08(0.29)$ & $0.91(0.19)$ & 0.05 \\
Lateral excursion $(\mathrm{cm})$ & $1.43(0.29)$ & $1.17(0.18)$ & 0.004 \\
\hline
\end{tabular}

Data are mean (SD) unless otherwise indicated

LVMI, left ventricular mass index.

mean pressure difference (54 (14) $\mathrm{mm} \mathrm{Hg} v 47$ (12) $\mathrm{mm} \mathrm{Hg}, \mathrm{p}=0.14$ ), fractional shortening (38 (7)\% v $39(8) \%, p=0.64)$, or ejection fraction $(76(8) \% v 75(8) \%, \mathrm{p}=0.96)$.

However, EOA was significantly lower in symptomatic than in asymptomatic patients

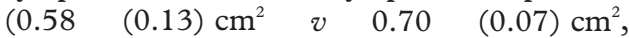
$\mathrm{p}=0.006)$. Long axis excursion was also lower in symptomatic than in asymptomatic patients, at both the septal $(0.91(0.19) \mathrm{cm} v 1.08(0.29)$ $\mathrm{cm}, \mathrm{p}=0.05)$ and the lateral sides of the annulus $(1.17(0.18) \mathrm{cm} \quad v \quad 1.43(0.30) \mathrm{cm}$, $\mathrm{p}=0.04$ ) (fig 3) (table 2). However, on multivariate logistic regression analysis, only long axis excursion at the lateral side of the mitral annulus $(p=0.004)$ and EOA $(p=0.02)$ were independently significant.

\section{Discussion}

This study shows that long axis excursion may be reduced in all grades of aortic stenosis even when fractional shortening and ejection fraction are normal.

\section{LEFT VENTRICULAR FUNCTION IN AORTIC STENOSIS}

An increase in afterload as a result of aortic stenosis causes a rise in myocardial wall stress. By the law of Laplace, wall stress is related directly to cavity size and inversely to wall thickness. Left ventricular hypertrophy, by causing an increase in wall thickness and a decrease in cavity size, tends to oppose the effect of the raised left ventricular pressure on wall stress. However, blood flow to the hypertrophied myocardium may be compromised leading to ischaemia and ultimately to fibrosis. Thus the left ventricular hypertrophy may initially be seen as an adaptive process, while ultimately leading to significant impairment of left ventricular systolic function.

The longitudinally or spirally arranged fibres in the subendocardial layers are responsible for the ventricle becoming more spherical during isovolumetric contraction. The intermediate circumferential fibres begin to contract a mean (SD) of 25 (40) ms later, and throughout the rest of systole both circumferential and longitudinal axes shorten synchronously. ${ }^{20}$ Most measures of systolic function including fractional shortening and ejection fraction predominantly describe transverse systolic function and reflect the activity of the circumferential fibres. However, the subendocardial fibres are at greater risk from ischaemia than the intermediate layers so that a decrease in the amplitude of long axis excursion precedes transverse dysfunction in patients with coronary artery disease, ${ }^{11}$ hypertension, mitral valve disease, and cardiomyopathies. ${ }^{12-14}$

This study shows that the same pattern occurs in aortic stenosis. Long axis excursion was lower in patients with even mild aortic stenosis $(1.2 \mathrm{~cm})$ than in the normal comparison group $(1.5 \mathrm{~cm})$. It was lower in those with moderate $(1.1 \mathrm{~cm})$ and severe $(1.0 \mathrm{~cm})$ stenosis in the presence of preserved fractional shortening, and was $0.8 \mathrm{~cm}$ in the group with established transverse dysfunction. The implication is that there is a progressive decline in left ventricular function as shown initially by long axis excursion and ultimately by measures of transverse function. This progression would, however, need to be confirmed by serial measurements in the same patients.

Reduced measures of systolic function in aortic stenosis can occur as a result of either myocardial fibrosis or increased wall stress. ${ }^{21} 22$ Relieving wall stress by replacing the stenotic valve allows some recovery of function, ${ }^{19}$ although intrinsic reductions in myocardial contractility as a result of fibrosis may not recover even several months after valve replacement. ${ }^{23}{ }^{24}$ Rediker and colleagues ${ }^{25}$ studied 42 patients with a left ventricular ejection fraction $<45 \%$ in the absence of coronary artery disease. Of these, 23 had serial gated nuclear ventriculography and nine $(39 \%)$ had a persistently abnormal ejection fraction after aortic valve replacement. Other studies have shown persisting left ventricular dysfunction in $24 \%$ to $66 \%$ of patients. ${ }^{23}{ }^{24}{ }^{26}$ In our study, long axis excursion was independently related to both left ventricular mass index and the grade of aortic stenosis. This suggests that both pathophysiological changes as a result of left ventricular hypertrophy and increased afterload as a result of aortic stenosis may together contribute to changes in systolic function in aortic stenosis. It is possible that long axis excursion is better able than conventional measures to predict the onset of impending irreversible changes in left ventricular function. $^{24}$

\section{PREDICTION OF SYMPTOMS}

The prognosis in symptomatic aortic stenosis is poor. There is a $50 \%$ mortality by three to five years in the presence of exertional chest pain, by two years with syncope, and by one year with overt heart failure. ${ }^{27}$ The outlook in asymptomatic aortic stenosis is widely regarded as too good to justify surgery ${ }^{3}$ although there is controversy about how frequently sudden death occurs. Furthermore, death can occur soon after the onset of symptoms, ${ }^{1}$ underlining the need for exceptionally careful follow up of asymptomatic patients. ${ }^{28}$ If a reliable method of predicting the onset of symptoms were available, this could guide the frequency of follow up. In countries where waiting times are long, investigation could be started prophylactically and, in selected patients, referral for prophylactic surgery could be considered. 
The grade of aortic stenosis is known to predict the onset of symptoms. Patients with a transaortic peak velocity $>4.0 \mathrm{~m} / \mathrm{s}$ have a $40 \%$ chance of developing symptoms each year, ${ }^{29}$ and we have confirmed that the EOA was lower in patients with symptomatic than in asymptomatic severe aortic stenosis. We also showed that reduced long axis excursion was independently related to the presence of symptoms. This is consistent with the observation of reduced coronary flow reserve $\mathrm{e}^{30}$ and abnormalities of coronary flow at rest ${ }^{31}$ in patients with symptomatic severe aortic stenosis. There was, however, substantial overlap in long axis excursion between patients with and those without symptoms so that its predictive ability remains uncertain and is being investigated in a prospective study.

\section{LIMITATIONS}

Long axis function may be affected by coronary disease as well as by aortic stenosis. This is unlikely to have affected our results because we excluded patients with regional wall motion abnormalities on the echocardiogram, and the presence of coronary disease did not emerge as a significant determinant of long axis excursion. Our normal comparison group was matched for age but not sex. However, this is unlikely to have affected our results because no sex differences in long axis function are reported. It would have been useful to estimate wall stress. However, we had no adequate estimate of left ventricular cavity pressures because most patients did not have cardiac catheterisation and, in those who did, the valve was rarely crossed.

\section{CONCLUSION}

In patients with aortic stenosis, long axis excursion may be abnormal even in the presence of normal fractional shortening and ejection fraction. Long axis excursion is inversely related to the grade of stenosis and is lower in symptomatic than in asymptomatic patients with severe aortic stenosis. This suggests the possibility that long axis excursion might be of use in predicting the onset of symptoms or of conventional measures of left ventricular dysfunction.

1 Pellikka PA, Nishimura RA, Bailey KR, et al. The natural history of adults with asymptomatic, hemodynamically sig-
nificant aortic stenosis. $\mathcal{F}$ Am Coll Cardiol 1990;15:1012-7.

2 Prendergast BD, Banning AP, Hall, RJC. Valvular heart disease. Investigation and management. Recommendations of a working group of The British Cardiac Society and the research working group of The British Cardiac Society and the research unit of the Royal College of

3 ACC/AHA guidelines for the management of patients with valvular heart disease. A report of the American College of Cardiology/American Heart Association. Task force on practice guidelines (committee on management of patient with valvular heart disease). 7 Am Coll Cardiol 1998;32 1486-588.

4 Lund O, Nielsen TT, Emmertsen K, et al. Mortality and worsening of prognostic profile during waiting time fo valve replacement in aortic stenosis. Thorac Cardiovasc Surg 1996;44:289-95.

5 Turina J, Hess O, Sepulcri F, et al. Spontaneous course of aortic valve disease. Eur Heart f 1987;8:471-83.

6 Lindblom D, Lindblom U, Qvist J, et al. Long-term relative survival rates after heart valve replacement. $\mathscr{f} \mathrm{Am}$ Coll Cardiol 1990;15:566-73.
7 Kelly TA, Rothbart RM, Cooper CM, et al. Comparison of outcome of asymptomatic to symptomatic patients older than 20 years of age with valvular aortic stenosis. Am $\mathcal{F}$ Cardiol 1988;61:23-30

8 Schulman DS, Remetz MS, Elefteriades J, et al. Mild mitral insufficiency is a marker of impaired left ventricular performance in aortic stenosis. F Am Coll Cardiol 1989;13: 796-803.

9 Brener SJ, Duffy CI, Thomas JD, et al. Progression of aortic stenosis in 394 patients: relation to changes in myocardial and mitral valve dysfunction. Progression of aortic stenosis in 394 patients: relation to changes in myocardial and mitral valve dysfunction. $f$ Am Coll Cardiol 1995;25:30510.

10 Kennedy KD, Nishimura RA, Holmes DRJ, et al. The natural history of moderate aortic stenosis. $\mathcal{F} \mathrm{Am}$ Coll Cardiol 1991;17:313-9.

11 Henein MY, Priestley K, Davarashvili T, et al. Early changes in left ventricular subendocardial function after successful coronary angioplasty. Br Heart f 1993;69:501-6.

2 Henein MY, Gibson DG. Suppression of left ventricular early diastolic filling by long axis asynchrony. Br Heart $\mathcal{F}$ 1995;73:151-7.

13 Henein MY, Gibson DG. Abnormal subendocardial function in restrictive left ventricular disease. Br Heart 71994 ; 72:237-42.

14 Alam M, Hoglund C, Thorstrand C, et al. Atrioventricular plane displacement in severe congestive heart failure following dilated cardiomyopathy or myocardial infarction. f Intern Med 1990;228:569-75.

15 Collinson J, Henein MY, Flather M, et al. Valve replacement for aortic stenosis in patients with poor left ventricular function. Circulation 1999;100:II-1-II-5.

16 Sahn DJ, De Maria A, Kisslo J, et al. The committee on M-mode standardization of the American Society of Echocardiography: recommendations regarding quantitation in M-mode echocardiography: results of a survey of echocardiographic measurements. Circulation 1978;58: 1072-81.

17 Troy BL, Pombo J, Rackley CE. Measurement of left ventricular wall thickness and mass by echocardiography. Circulation 1972;45:602-11.

18 Vuille C, Weyman AE. Left ventricle 1: general considerations, assessment of chamber size and function. In Weyman AE. Principles and practice of echocardiography, 2nd ed. Philadelphia: Lea and Febiger, 1994:575-624.

19 Choy AM, Darbar D, Lang CC, et al. Detection of left ventricular dysfunction after acute myoacrdial infarctioncomparison of clinical, echocardiograhic, and neurohumoral methods. Br Heart f 1994; 72:16-22.

20 Jones CJH, Raposo L, Gibson DG. Functional importance of the long axis dynamics of the human left ventricle. $\mathrm{Br}$ Heart f 1990;63:215-20.

21 Krayenbuehl HP, Hess OM, Monrad ES, et al. Left ventricular myocardial structure in aortic valve disease before, intermediate, and late after aortic valve replacement. Circulation 1989;79:744-55.

22 Ross J Jr. Afterload mismatch in aortic and mitral valve disease: implications for surgical therapy. $7 \mathrm{Am}$ Coll Cardiol 1985;5:811-26

23 Hwang $\mathrm{MH}$, Hammermeister KE, Oprian C, et al. Preoperative identification of patients likely to have left ventricular dysfunction after aortic valve replacement. Participants in the veterans administration cooperative study on valvular heart disease. Circulation 1989;80:I65-76.

24 Connolly HM, Oh JK, Orszulak TA, et al. Aortic valve replacement for aortic stenosis with severe left ventricular dysfunction. Prognostic indicators. Circulation 1997;95: 2395-400.

25 Rediker DE, Boucher CA, Block PC, et al. Degree of reversibility of left ventricular systolic dysfunction after aortic valve replacement for isolated aortic valve stenosis. $A m \mathcal{F}$ Cardiol 1987;60:112-8

26 Morris JJ, Schaff HV, Mullany CJ, et al. Determinants of survival and recovery of left ventricular function after aortic valve replacement. Ann Thorac Surg 1993;56:22-9.

27 Ross JJ, Braunwald E. Aortic stenosis. Circulation 1968;38: 61-7.

28 Vaile JC, Griffith MJ. Management of asymptomatic aortic stenosis: masterly inactivity but cat-like observation. Heart 1997;78:215-7.

29 Otto CM, Burwash IG, Legget ME, et al. Prospective study of asymptomatic valvular aortic stenosis - clinical, echocardiographic, and exercise predictors of outcome. Circulation 1997;95:2262-70.

30 Marcus ML, Donald BD, Hiratzka LF, et al. Decreased coronary reserve. A mechanism for angina pectoris in patients with aortic stenosis and normal coronary arteries. $N$ Engl F Med 1982;307:1362-7.

31 Omran H, Fehske W, Rabahieh R, et al. relation between symptoms and profiles of coronary artery blood flow velocities in patients with aortic valve stenosis: a study using transoesophageal Doppler echocardiography. Heart 1996;75:377-83. 